\title{
Quality-based technique for routing and playout buffering in IP Telephony over Mobile ad Hoc Network
}

\author{
Fabrizio Boi \\ MCLab, Department of Electronic Engineering \\ University of Cagliari \\ Cagliari 09123 Italy \\ f.boi@diee.unica.it
}

\author{
Luigi Atzori \\ MCLab, Department of Electronic Engineering \\ University of Cagliari \\ Cagliari 09123 Italy \\ I.atzori@diee.unica.it
}

\begin{abstract}
In the last few years, Voice over IP (VoIP) providers have been a strongly growth compared with traditional voice service operators. The main motivations are the low-cost of the packetbased technologies and the reliability of the current (wired) IP network. Nowadays a new network for VoIP applications have been placed: Mobile Ad hoc NETwork (MANET). We strongly believe that this technologies will have success in the next future. However this particular network includes different limitations one of these is the highly variability of the network topology and channel behavior. This is an obstacle to the service quality due to route losses and significant delay variations. In this our study, we want to present a new strategy to improve Quality of Service (QoS) in MANETs. Our strategy is divided in two tasks: selecting of transmission paths and adjusting the playout delay. In the first task we select the route that at the moment is characterized by low network delay and jitter through the use of QOLSR routing algorithm, while in the second task we use an adaptive intertalkspurt strategy founded on a objective quality-based approach as ITU-T E-Model. We also present preliminary results of the proposed strategy, which encourage further investigations.
\end{abstract}

\section{Categories and Subject Descriptors}

H.4.3 [Communications Applications] Computer conferencing, teleconferencing, and videoconferencing

\section{General Terms}

Algorithms, Experimentation.

\section{Keywords}

IP Telephony, MANET, OLSR, QOLSR, QoS, E-Model, playout buffering

\section{INTRODUCTION}

MANET networks are defined as dynamic multi-hop wireless temporary networks, which are established by a group of mobile hosts on a shared wireless channel [1]. The main characteristics of his particular type of network are the absence of a centralized system, all nodes communicate to every other and they are in

Mobimedia 2008 July 7-9, 2008, Oulu, Finland. Copyright 2008 ICST ISBN 978-963-9799-25-7/08/07 DOI 10.4108/ICST.MOBIMEDIA2008. 3981 continuous movement. On the one hand, these characteristics make these networks very attractive, since almost no wired installations are required making very easy the provisioning of the communication services; on the other hand, these make the deployment of reliable and high-quality services very challenging. In this context, a great role is taken by the routing algorithm

which is in charge of finding reliable routes among all the possible paths between the source and the destination, when network topology and channel performance change constantly. The proposed routing protocols can be classified in three categories $[2,3]$ : proactive, reactive and hybrid. The first builds routing tables exploiting a periodic exchange of topology information with other nodes in the network; the second determines routes only when needed; the last is a mix of other two protocols.

The latest developments have increased the service retain ability and accessibility in MANET network, which represent now a viable solution for the deployment of networking services for data application at low costs and minimizing network installations. However, many efforts are still needed to make this technology workable for streaming applications where QoS requirements, in terms of packet losses, delay and jitter, are more stringent than those characterizing data applications. Only few works appeared in the last years on the analysis of joint routing in real-time traffic transmission over MANET networks. [4] evaluates the performance of different reactive routing protocols, such as the Ad Hoc On Demand Distance Vector (AODV), Dynamic Source Routing (DSR) and Temporally-Ordered Routing Algorithm (TORA), when varying the load of real-time traffic. These routing protocols have an high transmission delay because they determine the routing table only if there is traffic in the network. In [5], the authors have analysed the performance of VoIP systems in an ad hoc network with stationery nodes when using two routing protocols: AODV and Optimized Link State Routing (OLSR). Also the authors in [6] have investigated the deployment of VoIP services with the AODV routing protocol and analysed different performances metrics, such as jitter, one-way delay, frequency of service interruptions and their duration. These are the important performance metrics that affect the service quality experience of the user of mobile VoIP phones. Both papers have highlighted that the available routing algorithms still need to be improved to support telephony services over mobile ad hoc networks at a satisfactory quality. On the basis of these observations, we have investigated the introduction of a new strategy for VoIP packets routing, which takes into account the ITU-T E-Model [7] to 
evaluate the quality perceived by the end-user. The resulting algorithm relies on the QOLSR extension of the OLSR protocol to allow the voice source to gather information about the network connectivity and transmission delays. Indeed, the QOLSR algorithm is used only to monitor the status of the network while the selection of the path is driven by the E-Model for conversational quality evaluation. To improve the robustness, the proposed algorithm also exploits network diversity so that more than one path can be exploited in parallel; in this case more copies of the same packets are sent through different independent paths. The selection of the most appropriate routes to the destination is performed according to an inter-talkspurt approach to minimize the impact of the service interruption on the voice quality. Additionally, routes selection is jointly performed with playout delay adjustments. These two tasks are performed together since we have observed that the setting of the playout delay heavily affects the benefit of the proposed routing strategy. In this paper we want to proposed a technique where highly variability of the network topology and channel behavior, which influences the service quality, are jointly addressed.

The paper is organized as follows. Section 2 provides an overview of past works, a description of the quality-based approach for adaptive de-jittering in VoIP communications and a description of the main features of the QOLSR protocol. The proposed strategy for joint routing and playout buffering is then described in section three. Section four provides the simulation results and section five draws final conclusions.

\section{VOIP IN MANET NETWORKS}

Only few past papers entirely addressed the subject of this paper, yet, specific issues in VoIP over MANETs have been quite analyzed in some past works, which we review in the following subsection. To better present the proposed solution, in the subsections 2.2 and 2.3 we also illustrate some relevant works dealing with de-jittering in wired networks and routing in MANETs.

\subsection{Overview in Wireless Mesh Networks}

The most common challenges against wireless VoIP are the high packet loss rates due to interference and fading in the wireless channels and excessive delay jitter caused by Carrier-Sense Multiple Access/Collision Avoidance (CSMA/CA) and retransmission mechanism. Such impairments are further magnified in a Wireless Mesh Network (WMN) environment. Significant works in the area of VoIP over one-hop wireless networks exist, whereas the literature on VoIP in WMNs networks, and in particular in MANETs, is limited. The difference between WMN and MANET is only the mobility of nodes in MANET, but the way to improve the QoS is similar. In [8] e [9], the authors analyzed end-to-end performance enhancements when introducing a packet aggregating approach. In particular, [8] proposes a concatenation mechanism to reduce VoIP protocol overhead in a real multihop mesh network. These researchers have created three different algorithms, which differ on the basis of where the aggregator is located: end-to-end, where all packets towards a common destination are aggregated at the ingress node only; hop-by-hop, where aggregation and de-aggregation is done at every node, leading to better aggregation possibilities at the expenses of higher complexity and delay with respect to the first approach; accretion algorithm, which is a mix of the previous two techniques. The main benefit corresponds to an increase in the number of calls that can be supported in the mesh network; however, the voice quality resulted to be affected by the aggregation. In [9], a different hop-by-hop aggregation mechanism is proposed. This technique significantly improves the performance of VoIP traffic meant as number of supported flows and at the same time reduces MAC layer busy time. But this mechanism are some drawbacks in terms of scalability; indeed, with only five hops the differences between packets noaggregation and aggregation traffic start to be low. Then, there aren't any analysis of perceived quality of these experimental study through the application of any existing quality model but also of channel traffic conditions as jitter, delay and packet loss ratio. Another approach to improve VoIP quality is to create differentiated services, which minimize starvation of best effort applications in the network [10]. [10] proposes the creation of Priority QoS Maps and indicates the difference such maps will have when compared to their Best Effort counterparts. By using Priority QoS Maps an end user can immediately decide where to move to in order to obtain the acceptable QoS their VoIP application, and his premium service, demands. However, in this paper there isn't any reference to a particular routing algorithm that may influence the composition of the Priority QoS Maps. In the [11], the authors made extensive experiments on a real WMN that supports VoIP, Internet and IPTV services. They have analyzed the performance in terms of delay and jitter at the increase of the service traffic load in the network. The result is that VoIP performs perfectly over five hops, but it has an higher worsening when there are any other type of traffic in parallel over the WMN as high definition IPTV stream or three standard definition IPTV channels in parallel only over one hop. [12] proposes an Adaptive QoS Playout (AQP) algorithm to offer a high quality wireless VoIP service. AQP integrates the effect of de-jitter buffer control, retransmission, and handoff delay based on a perceptual speech quality, the E-Model. AQP first configures several buffer delays and test their packet loss rate at the beginning of each talkspurt. Then, it evaluates the E-Model quality index of these chosen delays, from which it selects a good playout delay to optimize the quality of a VoIP session. The good idea of the AQP algorithm is, the consideration of the retransmission of voice packet to evaluate error recovery in improving voice quality. Anyway the values of R-Factors aren't good, indeed R is around 63 and it's a low quality of voice rating. On top of that, when the end-to-end delay is large, AQP performs similarly to existing adaptive algorithm and in this study there aren't another type of traffic in the WMN.

All of these papers analyze a part of the problem of VoIP QoS in a WMN and no one focus this research on MANET. In this paper we want to proposed a technique where highly variability of the network topology and channel behavior, which influences the service quality, are jointly addressed.

\subsection{Quality-based de-jittering}

The removal of transmission jitter in streaming applications is accomplished at the receive side by means of a playout buffer that masks this impairment at the expense of an additional delay. Within this framework, an important task is the setting of the total end-to-end delay, which should consider the network delay, the packet loss, and the perceived subjective quality. Originally, the 
setting of the playout buffer was purely based on the introduced additional delay and loss performance. In the last years, a different approach has been proposed, which consists in taking into account the effects of delay and losses on the subjective quality [13]. Such an approach requires the use of an appropriate tool to evaluate the combined effects of transmission impairments that affect the conversational quality. On the basis of this tool, the playout buffering algorithm estimates the optimal buffer configuration by weighting the contribution of delay and loss to the conversational quality. The use of such a perceptually motivated optimality criterion allows the receiver to automatically balance packet delay versus packet loss. The buffer size is adaptively adjusted so that the expected quality during the next conversation period is maximized. Almost all the proposed works founded on this approach, which we refer to with quality-based, make use of the ITU-T E-Model for quality evaluation [7], [14]. It is a computational framework for the estimation of the conversational quality by means of a synthetic index (the $\mathrm{R}$ Factor), which encloses the contributions of many features, presented as impairment factors. We briefly summarize the EModel, which provides an output index, called R-Factor, which measures the user-perceived quality:

$$
R=100-I_{s}-I_{d}-I_{e, e f f}+A
$$

$I_{S}$ includes all the impairments introduced in the circuitswitched part of the end-to-end communication path. $I_{d}$ mostly comprises the effects due to delay and echo. $I_{e, e f f}$ takes mainly into account the impairments caused by low bit-rate codecs and information loss. The expectation factor A raises the conversational quality when the end-user may accept some decreases in quality for access advantage (e.g., mobility). However, under some common assumptions these parameters can be simplified as follows [14]:

$$
\left\{\begin{array}{l}
I_{s}=6.8 \\
I_{d}=0.024 d_{e 2 e}+0.11\left(P_{D}-177.3\right) H\left(P_{D}-177.3\right) \\
I_{e, e f f}=11+40 \ln (1-10 E) \\
A=0
\end{array}\right.
$$

where $P_{D}$ is the total end-to-end delay (in ms), $E$ is the total packet loss rate, and $H(x)$ is the step function $(H(x)=0$ if $x<0$ and $H(x)=1$ otherwise). The R-Factor is then a function of the total packet loss rate and the end-to-end delay, named also playout delay. It's most important to note that the amount of losses depend on the playout delay too: the higher the delay is, the lower the loss rate is. The output index is defined in the range $0-100$, with higher values meaning higher service quality.

\subsection{Routing Algorithm}

To implement the proposed strategy, a routing protocol that allows the source to know the network topology and the link transmission delays is needed. We haven't defined any new routing protocol, but we have evaluated the plethora of existing ones and selected that which can provide this information with only minor changes, that is the OLSR protocol and its evolution QOLSR. Both these protocols, called proactive protocols, maintain routes to all destination at all times through periodic advertisements.
OLSR [15] is a table-driven proactive protocol which builds routing tables exploiting a periodic exchange of topology information with other nodes in the network. To create the routing table, this algorithm used two particular control messages called Hello and Topology Control (TC) messages. Hello messages are used by each node to communicate its position to the nodes of only 1-hop and are sent in broadcast only to the neighbour nodes. Each node selects a sub-set of its neighbour nodes of only 1-hop. These sub-set nodes, called Multi Point Relay nodes (MPR), are selected to announce the position periodically of its neighbour to other neighbouring nodes through their TC messages. Only a subset of all nodes are selected as MPR to reduce the number of nodes flooding link-state information as well (Fig. 1).
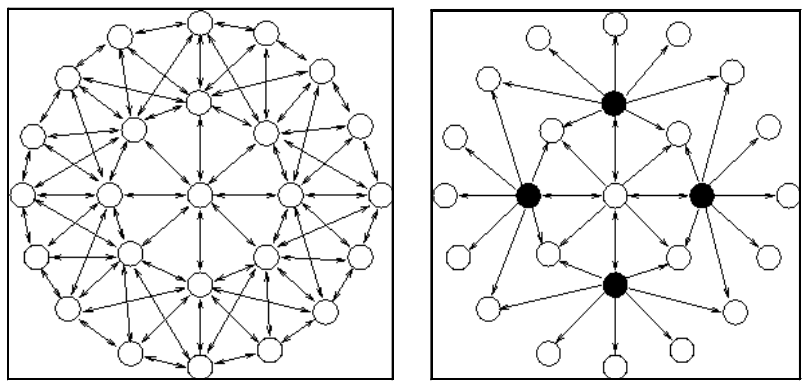

Fig. 1 (left) Traditional routing and (right) OLSR routing

QOLSR is an enhancement of the OLSR routing protocol to support multiple-metric routing criteria. Each node calculates various quality metric (delay, bandwidth, loss probability, etc) in every neighbour link, through the transmission of Hello messages and its relative timestamps. Then, all metrics are included in TC messages and sent to the other nodes to inform them. Moreover, metric informations are used to calculate sub-set nodes called QoS Multi Point Relay nodes (QMPR). Another difference between the two protocol versions is path selection: OLSR selects the shortest path considering the number of hops, whereas QOSLR selects the shortest paths on the basis of more metrics (delay, bandwidth, loss probability, etc).

\section{JOINT ROUTING AND PLAYOUT}

In the proposed algorithm, the voice source performs the most important operations. These consist in collecting information on the status of the MANET network and adaptively computing the best routes and playout delays. These tasks are accomplished in parallel and continuously during the voice communication session. However, the adjustments of the routes and the playout delays are performed intra-talkspurt, that is, these are changed only during silence periods to reduce the impact of voice segments shrinking and/or expansion on the service quality. We describe these main operations in the next three subsections.

\subsection{Overview of the proposed scheme}

As already anticipated in the previous section, in this paper we refer to the MANET network technology and implementation of IP Telephony services on top of it. The main characteristic of the MANET is the absence of a centralized node managing the network communications; indeed, this network is set up by mobile 
wireless peer nodes which may act as either source/destination or router in each communication. In this paper we refer to a particular MANET model called flat topology [16]. This topology is characterized by the complete absence of any centralize system; then, all nodes have equivalent responsibility and all of these contribute to find radio link to communicate, at least. Accordingly, it's possible that communications between two nodes are routed through different routes. One of benefits of this particular topology is the opportunity of setting one or more routes together in a end-to-end communication, and then it's possible to choose links depending on a particular criterion, as proposed in this devised algorithm.

VoIP applications have been developed over a set of protocols (RTP, UDP, and IP) that are not able to natively guarantee the application required quality of service. In fact, different factors deeply affect the end-user perceived quality. One of the most impairing factors is the variation of the packet transmission delay during the streaming, named jitter, which is caused by the temporal variability of the network conditions. Due to the high variability of the network topology and channel behavior, this impairment is even more annoying in MANET networks than in other types of network (wired or infrastructure wireless networks). We propose to reduce the extension of this impairment by monitoring the network status and selecting the route that at the moment is characterized by low network delay and jitter. To improve the resulting quality, we also consider sending voice packets over more paths in parallel so as to increase the probability to receive the packet in time before its playout instant. Additionally, since the quality of a route selection depends on the playout delay, we propose to control both route selection and playout buffer size jointly. This increases the effectiveness of both actions. The selection of the optimal configuration is performed on the basis of the ITU-T conversational quality model. It has the advantage to consider the most important network settings and performance metrics and to evaluate their impact on the voice quality taking into account the human perception. While this quality-based approach has been already proposed in the past for playout buffering, it is new in driving the paths selection.

\subsection{Statistics collection and processing}

The OLSR protocol provides the functionalities to allow each node to gather information about network connectivity. Since we also need delays statistics to evaluate the expected end-to-end quality of service, we assume that in the network is running the QOLSR protocol, which allows the nodes to exchange information on the quality metrics send additional information (e.g., delay, bandwidth). In our scenario we assume that every $T$ seconds, each node sends in broadcast different Hello messages both to communicate with other 1-hop nodes its position and the transmission delays. In this way each node acquires information about the local connectivity. To widespread this information through the entire network, the routing algorithm sends in broadcast TC messages by the QMPR nodes. This message contains the list of nodes directly connected to the QMPR and their quality metrics, in our study we consider only delays of each directly connected one. Therefore, every node is able to build the whole graph of the network. Since QOLSR is a proactive protocol, each change is trigger an update of the topology information. In our algorithm we have introduce a new feature to estimate the delays. Hello messages are used to convey information on link transmission delays and to create the oriented network graph. The setting of the $T$ parameter is very important to control the temporal resolution of the transmission delay statistics (the lower the value of $T$ the higher the resolution) and the traffic overhead (the higher the lower the amount of additional traffic).

\subsection{Path selection and playout delay setting}

Fig. 2 shows the flow graph of the proposed algorithm. Network connectivity and transmission delay monitoring, as described in the previous subsection, is performed during both talkspurt and silence periods and requires the cooperation of every node in the network.

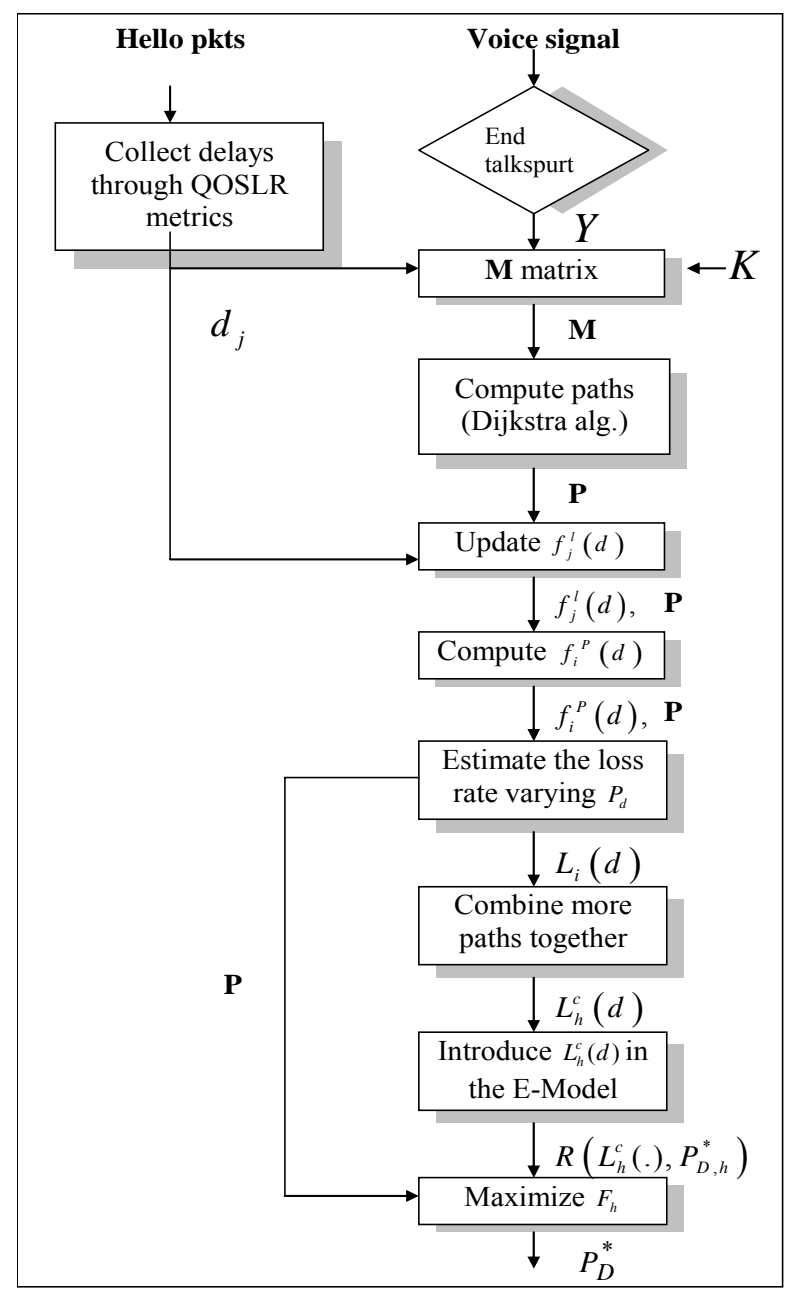

Fig. 2 Flow graph of our proposed algorithm.

Differently, path selection and playout delay setting is performed only during silence periods. There are many silence detection algorithms that can be used and that are implemented in most codecs to reduce the final source bit rate. As already mentioned, the proposed strategy exploits path diversity by sending more copies of voice packets along different paths in parallel when this is expected to have a significant positive impact on the voice 
conversational quality. The selection of the optimal paths in terms of the foreseen conversational voice quality, is performed on the basis of a plethora of preliminarily selected paths $\mathbf{P}$. To obtain the paths in $\mathbf{P}$, we compute the matrix $\mathbf{M}$, that represents the topology of the network, on the basis of the delays collected during the $K$ previous seconds of time over the entire network. This matrix is computed by the source averaging the delays observed over all the links in the network. This matrix then provides also information on the connectivity of the nodes since if no delays statistics have been collected during this interval in a link, then the relevant nodes are assumed not to be directly connected. We have then applied the Dijkstra's algorithm to matrix $\mathbf{M}$, which is a greedy algorithm that solves the single-source shortest path problem for a directed graph with non negative edge weights. The Dijkstra's algorithm is initially used to compute the shortest path; then, the relevant links are removed from matrix $\mathbf{M}$ and the algorithm is again applied.

These steps are performed iteratively, so as to obtained $N$ independent paths that are then stored in matrix $\mathbf{P}$ An example of matrix $\mathbf{P}$ is represented in Table 1, where the number of nodes of the network is $N=5$, obviously matrix $\mathbf{P}$ have dimension $N^{*} N$. In this matrix the independent paths are described by them relative weights $\mathrm{d} 1, \mathrm{~d} 2, \mathrm{~d} 3$, etc. This weight is calculated through the use of Dijkstra's algorithm. If there aren't any connection between two nodes, the relative weight is $\infty$. At this point, we need to elaborate the delay statistics collected up to now (from the beginning of the voice session) to estimate/update the probability density function (pdf) of the transmission delays observed in the links used in the paths in $\mathbf{P}$.

Table 1. An example of matrix $P$

\begin{tabular}{|c|c|c|c|c|c|}
\hline & Node1 & Node2 & Node3 & Node4 & Node5 \\
\hline Node1 & $\infty$ & $\infty$ & $\infty$ & $\mathrm{d} 2$ & $\mathrm{~d} 3$ \\
\hline Node2 & $\infty$ & $\infty$ & $\mathrm{d} 4$ & $\infty$ & $\infty$ \\
\hline Node3 & $\infty$ & $\mathrm{d} 4$ & $\infty$ & $\mathrm{d} 1$ & $\infty$ \\
\hline Node4 & $\mathrm{d} 2$ & $\infty$ & $\mathrm{d} 1$ & $\infty$ & $\infty$ \\
\hline Node5 & $\mathrm{d} 3$ & $\infty$ & $\infty$ & $\infty$ & $\infty$ \\
\hline
\end{tabular}

Let $j$ index these links and $f_{j}(d)$ the relevant pdf. There are several algorithms that can be used to build and update $f_{j}(d)$ from historical data; effective solutions are presented in [17]. In this work, we make use of the flush and refresh approach; however, more complex techniques that takes into account the age of the collected statistics can be used to improve the pdf prediction accuracy. $f_{j}(d)$ are then used to computed $f_{i}{ }^{P}(d)$, which is the pdf of the delays trough path $i$ in $\mathbf{P}(i=1, \ldots, N)$. Each $f_{i}{ }^{P}(d)$ is obtained by computing the convolution of all the $f_{j}(d)$ relevant to the links in path $i$. The result of the convolution is a good estimation of the pdf of the path delays as far as the delays encountered by a packet from a link to another are independent. Indeed, while this may be true in many scenarios, there are some circumstances that can affect this assumption (e.g., frequent collisions causes high transmission delays in adjacent wireless links).

The following operation is the estimation of the loss rate function $L_{i}(d)$ for every path $i$ varying the playout delay in a range such that the loss rate varies from 0 to 0.01 . We also consider all the possible combinations of paths that can be used to send copies of the same packets in parallel. We index all the possible combinations with $h(h=1, \ldots H)$, where $H$ is the maximum number of combinations made of $1,2,3, . ., N$ paths together. Note that $H$ includes also the $N$ single path solutions for presentation convenience. We then compute $L_{h}^{C}(d)$, which is the expected packet loss rate for any solution $h$. When more copies of the same packet are sent through more paths, the loss rate is obtained considering the probability that none of the copies sent in parallel arrives before the playout delay. The loss rate functions are then used in the E-Model to evaluate the performance expected when using each single path and any of paths combination. For any one of these possible solutions, we compute the playout delay that brings to the maximum R-Factor value: $P_{D, h}^{*}$. While the use of more paths together allows for higher R-Factor scores, we take into account the cost associated to the number of paths used with function $C$ (\#_copies). Let $c_{h}$ be the number of paths in combination $h$, we compute the cost for every solution as follows:

$F_{h}=R\left(L_{h}^{c}(),. P_{D, h}^{*}\right)+C\left(c_{h}\right)$

and we select the combination $h^{*}$ which maximizes (3). The path(s) in solution $h^{*}$ are then used during next talkspurt and the playout delay is changed to $P_{D, h^{*}}^{*}$.

\section{EXPERIMENTS}

We have performed extensive experiments using the Network Simulator 2 (NS2 v.2.29) environment with the additional OLSR protocol and the required changes to simulate the QOLSR protocol behavior [18]. We considered the MANET IEEE $802.11 \mathrm{~b}(11 \mathrm{Mbps})$ standard and simulated a wireless network in an area of $400 \mathrm{~m} \times 400 \mathrm{~m}$ with 10 nodes. Each node moves at the average speed of $5 \mathrm{~km} / \mathrm{h}$ in a flat country without any type of obstacle; each node generates background traffic directed to other 3-5 randomly selected stations with an overall rate of $500 \mathrm{kbps}$ with IP packets of 500 Byte on average and exponential interarrival time. Moreover the interested nodes generate Hello packets (any $1 \mathrm{sec}$ ) and TC messages (any $5 \mathrm{sec}$ ) to create the routing table.

All simulation runs last $200 \mathrm{sec}$ and the $\mathbf{M}$ matrix is updated considering the 5 previous seconds $(k=5 \mathrm{sec})$. Table 2 summarizes the main settings.

Once the algorithm has generated the weight graph and the $\mathbf{M}$ matrix, it selects $N=5$ shortest paths through the use of Dijkstra's algorithm (P matrix) but we consider at most 3 paths in parallel. It is not very convenient from the bandwidth point of view to use more than three paths in parallel because in these case we will only overload it. It's obvious that combinations of paths are double or triple. 
Table 2. Main settings

\begin{tabular}{|c|c|}
\hline \# nodes & 10 \\
\hline Working Area & $400 \mathrm{~m} \times 400 \mathrm{~m}$ \\
\hline MAC type & $802.11 \mathrm{~b}$ \\
\hline Simulation time & $200 \mathrm{sec}$ \\
\hline Hello packets interval (T) & $1 \mathrm{sec}$ \\
\hline TC packets interval & $5 \mathrm{sec}$ \\
\hline Background traffic rate & $500 \mathrm{kbps}$ \\
\hline Background packets dimension & $500 \mathrm{~B}$ \\
\hline
\end{tabular}

We have evaluated the performance of several traces in different conditions like different cost functions, different background traffic and so on. Now we want to present one of these in which we have compared different techniques:

- application of the OLSR algorithm combined with the quality-based playout control algorithm (we refer to this solution with OLSR);

- $\quad$ proposed algorithm without path diversity (we refer to this solution with Single Path);

- $\quad$ proposed technique with path diversity (we refer to this solution indicating the value of the cost function when using two or three paths in parallel).

Figs. 3 and 4 show the results of the three techniques at varying setting of the cost function. The enhancements of our algorithm are substantive both in R-Factor values and in playout delay. In the case of multiple paths, R-Factor reaches high values that allow to obtain good quality of voice conversation with low $P_{D}$ values. Though cost function can be higher the multiple paths solution detains about 10 points of R-Factor difference respect to single paths or OSLR solutions (Table 3).

Table 3. Playout delay and R-Factor: average and variance

\begin{tabular}{|l|c|c|c|c|}
\hline & $\begin{array}{c}\text { mean } \boldsymbol{D} \\
(\mathbf{s e c})\end{array}$ & $\boldsymbol{\sigma}_{D}^{2}$ & $\begin{array}{c}\text { mean } \boldsymbol{R} \\
(\mathbf{s e c})\end{array}$ & $\boldsymbol{\sigma}_{R}^{2}$ \\
\hline MultiPath C=0 & 0.212 & 0.00016 & 72.52 & 5.607 \\
\hline $\begin{array}{l}\text { Multi Path C(2)=3; } \\
\text { C(3)=6 }\end{array}$ & 0.218 & 0.00019 & 71.15 & 5.861 \\
\hline $\begin{array}{l}\text { Multi Path } \\
\text { C(2)=13; C(3)=16 }\end{array}$ & 0.247 & 0.00154 & 65.24 & 38.54 \\
\hline Single Path & 0.273 & 0.00391 & 53.32 & 101.6 \\
\hline OLSR & 0.397 & $\sim 0$ & 51.67 & 0.004 \\
\hline
\end{tabular}

If the cost function grows considerably, R-Factor have many ripples (R-Factor variance very high); this is a particular characteristic of our technique because in this case it prefers to select a single path or two paths at a time but never more, then the enhancements of the multi paths decrease. OSLR algorithm have constant values both of R-Factor and $P_{D}$ but there are not very good values. The constance is owing to the absence of any technique based on QoS but also to investigation of the minimal path with minimum hops.

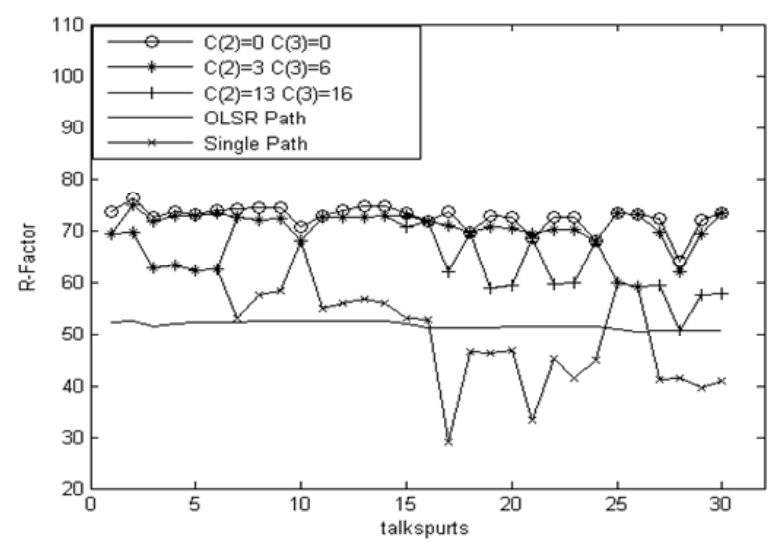

Fig. 3 R-Factor: comparison of alternative techniques.

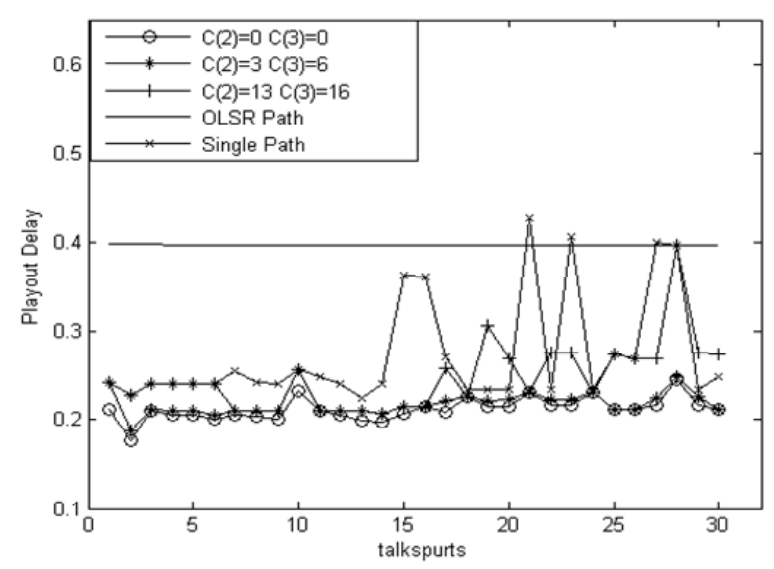

Fig. 4 Playout delay: comparison of alternative techniques

\section{CONCLUSIONS}

We have presented a new strategy for route selections and playout control for VoIP services in MANET networks. The major idea is to consider at the same time different and independent paths with different copies of the same packet such that if one or more mobile nodes are suddenly down there is an alternative path to guarantee the communication and the quality to the end user. This approach improves the end user perceived quality by the utilization of E-Model and by maximization of R-Factor in the selection of the independent paths. The performed tests show good improvements of quality and moderate playout delay values without more and heavy variations. Future work is aimed to investigating new setting of parameter to improve further on quality of the voice communication.

\section{ACKNOWLEDGMENTS}

This research activity is partially funded by the IKNOS project funded by the Italian Ministry of education and research and developed by the University of Cagliari, CNIT and Tiscali. 


\section{REFERENCES}

[1] Toh, C. K. 1997. Wireless ATM and Ad Hoc Networks: Protocols and Architecture. Kluwer Academic Publishers, Dordrecht, 1997.

[2] Scholten, H., Jansen, P.G., and Hop, L. 2004. Routing in wireless multimedia home networks. ICME (Taipei, 2004), 1799-1802.

[3] Zou, X., Ramamurthy, B., and Magliveras, S. 2002. Routing Techniques For Wireless Ad Hoc Networks-Classification and Comparison. Proceedings of Sixth World Multiconference on Systemics, Cybernetics and InformaticsSCI (July 2002).

[4] Lee, K. S., Lee, S. J., and Chung, Y. K., 2005. A Performance Comparison of On-Demand Routing Protocols for Application Data Mobile Ad hoc Networks. SERA, 331339.

[5] Armenia, S., Galluccio, L., Leopardi, A., and Palazzo, S., 2005. Transmission of VoIP Traffic in Multihop Ad Hoc IEEE 802.11b Networks: Experimental Results. WICON, $148-155$

[6] Thibodeau, E., Youssef, M., and Houle, A. C., 2006. Investigating MANET performance in a VoIP context. IEEE CCECE/CCGEI (May 2006), 920 - 923.

[7] ITU-T Recommendation G.107, 2003. The E-Model, a computational model for use in transmission planning. (Marzo 2003).

[8] Kim, K., Ganguly, S., Izmailov, R., Sangjin Hong, 2006. On Packet Aggregation Mechanisms for Improving VoIP Quality in Mesh Networks. Vehicular Technology Conference (VTC 2006-Spring, 7-10 May 2006). IEEE 63rd Vol. 2, 891-895.

[9] Castro, M C., Dely, P., Karlsson, J., Kassler, A., 2007. Capacity Increase for Voice over IP Traffic through Packet Aggregation in Wireless Multihop Mesh Networks. Future Generation Communication and Networking, Vol. 2, 350 355 .
[10] Dao, N.T., Malaney, R.A., Exposito, E., Xun Wei, 2005. Differential VoIP service in Wi-Fi networks and priority QoS maps. Global Telecommunications Conference 2005 (GLOBECOM '05, Dec. 2005), IEEE Vol. 5, 2653 - 2657.

[11] Ekling, J., Gidlund, M., Flodin, R., 2007. Performance of Triple Play Services in Wireless Meshed Networks. Consumer Electronics (ISCE , 20-23 June 2007), IEEE International Symposium, 1-5.

[12] Liang-Yi Huang, Yung-Mu Chen, Tein-Yaw Chung, ChihHung Hsu, 2007. Adaptive VoIP Service QoS Control based on Perceptual Speech Quality. Advanced Communication Technology (The 9th International Conference on Volume 2, 12-14 Feb. 2007), $885-890$.

[13] Atzori, L., and Lobina, M.L., 2006. Playout Buffering in IP Telephony: a Survey Discussing Problems and Approaches. 36-46

[14] Cole, R., and Rosenbluth, J., 2001. Voice Over IP performance monitoring. ACM Computer Communication Review (April. 2001), Vol. 31, no. 2.

[15] Clausen, T., and Jacquet, P., 2003. Optimized Link State Routing Protocol. IETF Internet Draft, draft-ietf-manet-olsr11.txt.

[16] Ramachandra, K., and Ali, H.H., 2004. Evaluating the performance of various architectures for wireless ad hoc networks. Proceedings of the 37th Annual Hawaii International Conference.

[17] Sreenan, C.J., Chen, J.C., Agrawal, P. and Narendran, B., 2000. Delay reduction techniques for playout buffering. IEEE Trans. on Multimedia (June 2000), Vol. 02, no. 02, 88100 .

[18] The TUCB/LBNL/VINT Network Simulator. http://www.mash.cs.berkeley.edu/ns. 